\title{
REDE DE ATENÇÃO ÀS URGÊNCIAS E EMERGÊNCIAS: PERFIL, DEMANDA E ITINERÁRIO DE ATENDIMENTO DE IDOSOS*
}

\author{
Bárbara Cris Skora Antunes ${ }^{1}$, Karla Crozeta ${ }^{2}$, Fernanda de Assis ${ }^{3}$, Maria Cristina Paganini ${ }^{4}$
}

RESUMO: Objetivo: caracterizar o perfil, a demanda e o itinerário de idosos cadastrados em uma Unidade Municipal de Saúde, atendidos na Rede de Atenção às Urgências e Emergências de Curitiba. Método: pesquisa avaliativa, documental, transversal, de abordagem quantitativa, realizada entre agosto de 2015 e junho de 2016, cuja fontes de dados foram Relatórios de Atendimento na Rede e prontuários eletrônicos de usuários com 60 anos ou mais. A análise foi realizada por estatística descritiva. Resultados: identificaram-se 102 idosos que realizaram 160 consultas na Unidade de Pronto Atendimento, 59 (57,8\%) eram do sexo feminino, 50 (49\%) tinham entre 60 e 69 anos, em 55 (53,9\%) a demanda foi considerada "pouco urgente", e 10 foram internados. Conclusão: observou-se a demanda de usuários devido a agravos comuns de saúde, sendo necessária a reorganização operacional e gerencial da rede, objetivando adequação do atendimento em todos os seus níveis de complexidade.

DESCRITORES: Enfermagem; Atenção primária à saúde; Serviços médicos de emergência; Idoso.

\section{URGENT AND EMERGENCY CARE NETWORK: PROFILE, DEMAND AND FLOW OF ELDERLY TO HEALTH CARE POINTS*}

ABSTRACT: Objective: characterize the profile, demand and flow of care to elderly registered in a Municipal Health Unit assisted at an Emergency Care Network (RUE) of Curitiba. Method: cross-sectional study of quantitative approach for assessment and documentation purposes conducted between August 2015 and June 2016, based on reports of care provided to users at the referred Network and electronic medical records of users aged 60 and over. Descriptive statistics was used in the analysis. Results: it was found that 102 elderly people made 160 visits at the Emergency Care Unit (UPA), as follows: 59 (57.8\%) were women, 50 (49\%) were aged 60-69 years old, the demands of $55(53.9 \%)$ were considered to be "not very urgent" and 10 individuals were hospitalized. Conclusion: analysis of the demands of users with ordinary health problems indicated the need for operational and managerial reorganization of the network, for adequacy of care at all its levels of complexity.

KEYWORDS: Nursing, Primary health care; Medical emergency services; Elderly

\section{RED DE ATENCIÓN A URGENCIAS Y EMERGENCIAS: PERFIL, DEMANDA E ITINERARIO DE ATENDIMIENTO DE ANCIANOS*}

RESUMEN: Objetivo: caracterizar el perfil, la demanda y el itinerario de ancianos registrados en una Unidad Municipal de Salud, atendidos en la Red de Atención a Urgencias y Emergencias de Curitiba. Método: pesquisa evaluativa, documental, transversal, de abordaje cuantitativo, realizada entre agosto de 2015 y junio de 2016, cuyas fuentes de datos fueron Informes de Atendimiento en la Red y prontuarios electrónicos de usuarios con 60 años o más. El análisis fue realizado por estadística descriptiva. Resultados: se identificaron 102 ancianos que realizaron 160 consultas en la Unidad de Urgencias, $59(57,8 \%)$ eran del sexo femenino, 50 (49\%) tenían entre 60 y 69 años, en 55 $(53,9 \%)$ se consideró la demanda "poco urgente", y 10 fueron internados. Conclusión: se observó la demanda de usuarios a causa de agravios comunes de salud, siendo necesaria la reorganización operacional y administrativa de la red, con el objetivo de adecuar el atendimiento en todos sus niveles de complejidad.

DESCRIPTORES: Enfermería; Atención básica a la salud; Servicios médicos de emergencia; Anciano.

'Enfermeira. Graduada e Licenciada em Enfermagem. Residente em Urgência e Emergência no Hospital de Clinicas da Universidade Federal do Paraná. Curitiba-PR-Brasil.

${ }^{2}$ Enfermeira. Doutora em Enfermagem. Docente do Departamento de Enfermagem da Universidade Federal do Paraná. Curitiba-PRBrasil.

${ }^{3}$ Enfermeira. Mestranda em Enfermagem do Programa de Pós-graduação em Enfermagem - Mestrado Profissional, da Universidade Federal do Paraná. Curitiba-PR-Brasil.

${ }^{4}$ Enfermeira. Doutora em Enfermagem. Docente do Departamento de Enfermagem da Universidade Tuiuti do Paraná. Curitiba-PRBrasil.

Universidade Federal do Paraná

R. Lothário Meissner, no 3400, CEP 80210-170, Curitiba, Paraná, Brasil

E-mail: karla.crozetafigueiredo@gmail.com 


\section{INTRODUÇÃO}

São representativos os avanços alcançados pelo Sistema Único de Saúde (SUS) nesses 27 anos de história no Brasil. Porém, com a mudança no perfil da população e dos agravos de saúde, e com os obstáculos para consolidação dos princípios constitucionais do sistema, tornou-se evidente a dificuldade em superar a fragmentação intensa dos serviços e ações de saúde, e a necessidade em aumentar a qualidade, a eficácia e a integralidade da assistência no cenário de saúde atual ${ }^{(1-2)}$.

Nesse sentido, surgiram as Redes de Atenção à Saúde (RAS), que são arranjos de organização do sistema para integrar o cuidado, visando à efetividade, segurança e qualidade na resolução dos principais problemas de saúde da população brasileira( ${ }^{(2-3)}$.

No cenário de saúde brasileiro há predomínio de doenças crônicas não transmissíveis (DCNT). Constatou-se que nos dias atuais, os brasileiros apresentam alta morbimortalidade relacionada com as doenças do aparelho circulatório, principalmente infarto agudo do miocárdio (IAM) e de acidente vascular encefálico (AVE), sendo essas as principais causas de mortalidade entre homens e mulheres no país ${ }^{(4)}$.

Ao encontro das estatísticas do país, estima-se que, no ano de 2015, a principal causa de mortalidade dos curitibanos foram as doenças do aparelho circulatório, com aproximadamente $27,45 \%$ dos óbitos, seguida das neoplasias com $21,20 \%$ dos óbitos, e pelas causas externas que representam 12,14\% das causas de mortalidade na cidade ${ }^{(5)}$. Tais dados revelam que a atenção às urgências e emergências devem ser pautas fundamentais e prioritárias para o governo federal e para os diversos municípios do país, visto que os indivíduos são acometidos todos os dias por tais agravos, e a ausência ou inadequação de um sistema capaz de lidar com tais problemas pode gerar incapacidade, óbito ou sofrimento do indivíduo e de sua família ${ }^{(6-7)}$.

Visto a problemática e a necessidade de intervenção adequada, foram desenvolvidas as Redes de Atenção às Urgências e Emergências (RUE). Constituindo uma das principais temáticas das RAS, objetivam atender os agravos de saúde dos usuários do SUS na área de urgência e emergência de forma resolutiva e oportuna, articulando todos os equipamentos de saúde, ampliando e qualificando o acesso humanizado e integral ${ }^{(8)}$.

A RUE propõe o atendimento de diferentes condições, que variam desde as situações clínicas, cirúrgicas, traumatológicas e relacionadas com as causas externas, como a violência, até gineco-obstétricas, psiquiátricas e pediátricas. Assim, a rede visa à ampliação e articulação dos serviços de urgência, de forma qualificada e resolutiva, articulando ações de promoção da saúde e prevenção de doenças e agravos, diagnóstico, tratamento, reabilitação e cuidados paliativos ${ }^{(8)}$.

A Atenção Primária à Saúde (APS), um dos componentes da RUE e porta de entrada do usuário, tem como objetivo a ampliação do acesso, o fortalecimento de vínculo e o primeiro atendimento às urgências e emergências, em ambiente adequado, até a transferência ou encaminhamento do paciente aos demais pontos de atenção da rede, quando necessário, levando em consideração o acolhimento com avaliação de risco e vulnerabilidades. Somado a isso, a APS deve ser a base para o cuidado, ser resolutiva, coordenar o cuidado e ordenar a rede ${ }^{(8)}$.

Por todo o exposto, a realização do presente estudo foi motivada pela necessidade de conhecimento acerca do perfil e da demanda de atendimento na RUE dos pesquisadores, assim como do itinerário percorrido pelos usuários, identificando os principais agravos de saúde, permitindo a avaliação e o levantamento de dados, que podem ser úteis para a manutenção de ações nos diferentes serviços e pontos de atenção.

Nesse cenário, o presente estudo integrou as ações de pesquisa do Programa de Educação pelo Trabalho para a Saúde (PET Saúde) - Redes de Atenção Curitiba, com o objetivo de caracterizar o perfil, a demanda e o itinerário de idosos de uma Unidade Municipal de Saúde atendidos na Rede de Atenção às Urgências e Emergências. 
Trata-se de uma pesquisa avaliativa, documental, transversal e de abordagem quantitativa, realizada a partir de dados de usuários cadastrados em uma Unidade Municipal de Saúde (UMS) localizada na cidade de Curitiba - Paraná, que atua na Estratégia de Saúde da Família (ESF), a fim de caracterizar o perfil, itinerário e demanda dos atendimentos na RUE.

A fonte de dados do estudo foi constituída pelos Relatórios de Atendimentos nas Unidades de Pronto Atendimento (UPA), referência para o território da UMS, no período de janeiro a julho de 2015, os Relatórios de Internamentos nos Hospitais do período entre maio a julho de 2015, e os prontuários eletrônicos dos pacientes cadastrados na UMS.

A coleta de dados ocorreu no período de agosto a setembro de 2015, e foram incluídas as informações dos atendimentos de usuários com 60 anos de idade ou mais nos serviços da RUE de Curitiba, com cadastro definitivo na UMS, atendidos no período de janeiro a julho de 2015. Foram excluídos os dados dos usuários com cadastro provisório na UMS ou os que não possuíam dados de atendimento no prontuário eletrônico.

Os dados coletados foram tabulados em planilhas do programa Microsoft Excel 2013, e analisados descritivamente no programa Statistical Package for Social Science for Windows (SPSS) (distribuição de frequências, médias e medianas), apresentados em quadros e tabelas. Os resultados foram agrupados em duas categorias: atendimentos na UPA e atendimentos nos Hospitais.

As variáveis de análise dos atendimentos na UPA foram: idade, sexo, frequência de atendimentos na UPA (número de consultas realizadas no serviço), quantidade de atendimentos, diagnóstico conforme capítulo da Classificação Internacional de Doenças (CID-10), descrição completa do diagnóstico, classificação de risco (Protocolo de Avaliação e Classificação de Risco conforme Manchester), período da consulta, retorno na UMS em até sete dias, intervalo de tempo entre atendimento na UPA e Visita Domiciliar (VD) (período de dias entre o último atendimento na UPA e a primeira VD), atendimento com mesmo diagnóstico ou semelhante, e internamento sequencial.

$\mathrm{Na}$ análise dos internamentos foram considerados: a idade, o sexo,o estabelecimento de destino, o diagnóstico conforme CID, a realização de VD pela equipe de saúde e o período de tempo entre hospitalização e retorno na UMS após alta hospitalar.

O estudo foi submetido ao Comitê de Ética em Pesquisa do Setor de Ciências da Saúde da Universidade Federal do Paraná (CEP/SD/UFPR) e ao Comitê de Ética da Secretaria Municipal de Saúde (SMS) de Curitiba, sendo aprovado sob os pareceres número 499.507 e 547.439, respectivamente.

\section{RESUltados}

Ao analisar os Relatórios de Atendimento na UPA, foi possível identificar que 102 idosos cadastrados na UMS foram atendidos no período de estudo. Destes, 59 (57,84\%) eram do sexo feminino e 50 $(49,01 \%)$ possuíam faixa etária entre 60 e 69 anos de idade, conforme Tabela 1.

Tabela 1 - Faixa etária dos idosos atendidos na UPA. Curitiba, PR, Brasil, 2016

\begin{tabular}{lcc}
\hline Faixa etária & $\mathbf{N}$ & $\%$ \\
\hline 60 a 69 anos & 50 & 49,01 \\
\hline 70 a 79 anos & 43 & 42,15 \\
\hline 80 a 89 anos & 19 & 18,62 \\
\hline 90 a 99 anos & 2 & 1,69 \\
\hline 100 anos ou mais & 1 & 0,98 \\
\hline TOTAL & 102 & 100 \\
\hline
\end{tabular}


Dos 102 idosos, $69(67,64 \%)$ realizaram um atendimento e $33(32,35 \%)$ realizaram dois ou mais atendimentos. (Tabela 2). Ao total foram realizados 160 atendimentos na UPA em questão, totalizando uma média de 1,56 consultas por idoso.

Tabela 2 - Frequência de atendimentos dos idosos na UPA. Curitiba, PR, Brasil, 2016

\begin{tabular}{lcc}
\hline Número de atendimentos & $\mathbf{N}$ & $\mathbf{\%}$ \\
\hline 1 & 69 & 67,64 \\
\hline 2 & 16 & 48,48 \\
\hline 3 & 12 & 36,36 \\
\hline 4 & 4 & 12,12 \\
\hline 7 & 1 & 3,03 \\
\hline TOTAL & 102 & 100 \\
\hline
\end{tabular}

Ao que diz respeito ao Protocolo de Avaliação e Classificação de Risco, um idoso (0,98\%) apresentou um atendimento com a classificação "emergente" (vermelho), um (0,98\%) o atendimento foi "muito urgente" (laranja), 13 (12,74\%) apresentaram atendimento "urgente" (amarelo), 55 (53,92\%) atendimento "pouco urgente", quatro (3,92\%) apresentaram atendimento pelo SAMU, 21 (20,58\%) apresentaram atendimentos com diversas classificações, e sete $(6,86 \%)$ foram ignorados devido à falta do dado (Tabela $3)$.

No que se refere ao diagnóstico, conforme a CID-10, dos 102 idosos, 69 (67,64\%) apresentaram apenas um diagnóstico e 33 (32,35\%) apresentaram dois ou mais, visto que, realizaram múltiplos atendimentos na UPA. Desses 69 atendimentos, 22 (31,88\%) ocorreram devido a sintomas, sinais e achados anormais de exames clínicos e de laboratório, 14 (20,28\%) a fatores que influenciam o estado de saúde e o contato com os serviços de saúde, sete $(10,14 \%)$ a doenças do aparelho circulatório e $26(37,70 \%)$ referentes a outros diagnósticos que ocorreram com distribuição inferior a sete.

Em relação aos 33 idosos que realizaram múltiplos atendimentos, somando 91 consultas, 18 idosos $(54,54 \%)$ obtiveram dois ou mais diagnósticos iguais ou alocados no mesmo capítulo da CID e $15(45,46 \%)$ idosos obtiveram diagnósticos distintos em todos os atendimentos. Dos 18 idosos, seis $(16,67 \%)$ apresentaram em todos os atendimentos, diagnósticos diversos.

Desses diagnósticos, prevaleceram os sintomas, sinais e achados anormais de exames clínicos e de laboratório com frequência de $25(27,47 \%)$, seguidas das doenças do aparelho respiratório com 21 $(23,07 \%)$ casos, fatores que influenciam o estado de saúde e o contato com os serviços de saúde com $15(16,48 \%)$ casos, e doenças do aparelho circulatório com sete $(7,69 \%)$ casos. Os demais 67 casos ocorreram devido a outros agravos de saúde.

O período de tempo entre atendimento na UPA e a VD da equipe da ESF teve uma média de 22,85 dias. Dois idosos tiveram VD antes do último atendimento na UPA. Não foi possível identificar atendimento sequencial em todos os casos, visto a falta de tal informação nos prontuários eletrônicos.

No período de maio a julho de 2015, 10 idosos cadastrados na UMS foram internados. Desses, três $(30 \%)$ eram do sexo feminino e sete $(70 \%)$ eram do sexo masculino. Das três mulheres, todas possuíam atendimento anterior na UPA, e dos homens, apenas dois não possuíam atendimento anterior na UPA. Ao total, ocorreram 12 internamentos por diferentes CID, visto que dois idosos (20\%) possuíram dois internamentos em meses diferentes. Desses 12 internamentos, 8 (66,67\%) foram em hospitais e quatro $(33,33 \%)$ foram na UPA (Tabela 3$)$. 
Tabela 3- Doenças diagnosticadas nos idosos em internamentos $(n=12)$. Curitiba, PR, Brasil 2016

\begin{tabular}{lcc}
\hline Capítulo do CID & $\mathbf{n}$ & $\mathbf{\%}$ \\
\hline Algumas doenças infecciosas e parasitárias & 1 & 8,34 \\
\hline Doenças do aparelho circulatório & 1 & 8,34 \\
\hline Doenças respiratórias & 5 & 41,67 \\
\hline Doenças da pele e do tecido subcutâneo & 1 & 8,34 \\
\hline Doenças do aparelho geniturinário & 1 & 8,34 \\
\hline $\begin{array}{l}\text { Sinais, sintomas e achados anormais de exames clínicos e de laboratório, não classificados } \\
\text { em outra parte }\end{array}$ & 1 & 8,34 \\
\hline Lesões, envenenamentos e algumas outras consequências de causas externas & 2 & 16,67 \\
\hline TOTAL & 12 & 100 \\
\hline
\end{tabular}

Dos 12 internamentos, em um caso (8,33\%) foi possível identificar a data da alta hospitalar. O tempo de permanência do idoso no serviço de saúde foi de cinco dias. Dos 10 usuários, sete (70\%) receberam VD da equipe da ESF após todos os internamentos e três (30\%) não receberam. Em relação ao retorno na UMS após hospitalização, seis idosos (60\%) retornam após as hospitalizações e quatro (40\%) não retornaram.

\section{DISCUSSÃO}

Neste estudo foi possível analisar o perfil, as demandas e o itinerário de idosos cadastrados em uma Unidade Municipal de Saúde de Curitiba na RUE. Foram atendidos 102 idosos na UPA em um período de sete meses, gerando um total de 160 consultas no serviço.

As UPAs são estruturas de complexidade intermediária entre as unidades básicas de saúde (UBS) e os hospitais. Elas compõem uma rede organizada de serviços que visam atender as urgências e emergências, através de pactos e fluxos pré-definidos. Possuem como principal objetivo o acolhimento dos usuários, intervindo em sua condição clínica, garantindo a resolubilidade da atenção, por intermédio da contra referência para os demais pontos da rede de atenção, proporcionando continuidade e integralidade no tratamento ${ }^{(9)}$.

Em relação aos idosos, 57,84\% eram do sexo feminino e 49,01\% possuíam de 60 a 69 anos de idade. Em uma revisão integrativa que buscou identificar a produção de conhecimento sobre as características dos usuários frequentes nos serviços de urgência, identificou-se que estudos revelam que as mulheres são as usuárias mais frequentes. Além disso, os extratos etários mais avançados, de 65 anos ou mais, são as pessoas que mais recorrem aos serviços. ${ }^{(10)}$ Outro estudo observou que, dos 385 indivíduos que buscaram o serviço de urgência, 211 eram do sexo feminino e 142 possuíam entre 60 e 79 anos de idade. O predomínio do sexo feminino pode estar relacionado com o nível de preocupação das mulheres com cuidados à saúde ${ }^{(11-12)}$.

Em relação à frequência, $67,64 \%$ realizaram um atendimento e 32,35\% realizaram dois ou mais atendimentos. Estudos indicam que a reincidência do usuário pode variar de 4 a 58 vezes, porém, usualmente os pacientes são pouco assíduos no serviço, seguidos dos que recorrem de forma moderada a altamente frequente ${ }^{(12)}$.

Os idosos do estudo apresentaram queixas semelhantes. Como principal queixa de saúde, destacam-se os sintomas, sinais e achados anormais de exames clínicos e de laboratório, seguida dos fatores que influenciam o estado de saúde e o contato com os serviços, doenças do aparelho respiratório e circulatório, lesões, envenenamentos e algumas outras consequências de causas externas. Esta situação reflete a busca constante por problemas comuns de saúde, impactando na superlotação dos serviços de média e alta complexidade, dificultando a operacionalização da rede. 
Atualmente, no Brasil e no SUS, utiliza-se o Sistema de Triagem de Manchester (STM), que possui cinco categorias para risco: vermelho (emergência, atendimento imediato), laranja (muito urgente, atendimento em 10 minutos), amarelo (urgente, atendimento em 60 minutos), verde (pouco urgente, atendimento em 120 minutos) e azul (não urgente, atendimento em 240 minutos $^{(13)}$. A maioria dos atendimentos referentes ao capítulo 18 do CID que possuíram maior prevalência nos idosos, trata-se de quadros clínicos que não possuem um diagnóstico fechado; portanto, não se referem a uma patologia propriamente dita, mas, sim, a sintomatologias que requerem continuidade de atenção em outros serviços de saúde, como a UBS(14).

Das consultas da UPA, 53,92\% dos idosos tiveram classificação "pouco urgente" e 20,58\% possuíam diversas (principalmente "pouco urgente" e "urgente"). Estudos que buscaram identificar a demanda de idosos na UPA observaram que os usuários com 60 anos ou mais possuíam em 69,1\% dos casos necessidades de atenção inadequadas para resolução no serviço de urgência. Visto a grande procura de casos de baixa gravidade e de queixas não urgentes no serviço de urgência e emergência, conclui-se que a maioria das situações era passível de resolução na $\mathrm{APS}^{(9)}$.

Em muitas situações, como encontradas neste estudo, as UPAs têm se tornado "portas de entrada" no sistema de saúde, atendendo tanto situações de emergência como de atenção básica e especializada. Existem inúmeros motivos pela busca constante da UPA. Dentre eles, pode-se citar a baixa resolutividade das UBS, visto a limitada disponibilidade de especialistas; exames e medicamentos; dificuldade de acesso devido à marcação de consultas; filas longas e horários restritos de atendimento; maior aparato tecnológico; maior utilização dos serviços pela população de municípios menores;e percepção dos usuários de atendimento de forma rápida e fácil ao serviço de saúde ${ }^{(9)}$.

Nesse sentido, a relação da população com a Unidade Básica de Saúde (UBS) pode ficar comprometida. O que ocorre é que, muitas vezes, o usuário não consegue atendimento na sua unidade de saúde em tempo hábil e fica um longo período de tempo na fila de espera, aguardando uma consulta. Após conseguir atendimento, nem sempre o usuário retorna à UBS para realizar continuidade dos cuidados, exceto quando ele está inserido em algum programa específico ${ }^{(15)}$.

Uma ferramenta que auxilia a equipe de saúde nesse contexto é a visita domiciliar, considerado um importante instrumento na APS. É utilizada para inserção e conhecimento do contexto e dinâmica familiares, produzindo a criação de vínculo. Ainda, permite conhecer as reais necessidades de saúde, proporcionando aproximação com os determinantes do processo de saúde-doença. Dos dados disponíveis, dos 58 idosos, 40 receberam a visita domiciliar, principalmente do Agente Comunitário de Saúde (ACS). Estudo relatou números semelhantes em que a maioria dos pacientes que foi ao serviço de urgência recebeu visita da equipe de saúde posteriormente ${ }^{(16)}$.

Os idosos apresentaram 12 internações em um período de três meses, sendo oito em hospitais e quatro na UPA, tendo como principal diagnóstico as doenças respiratórias e as lesões, envenenamentos e algumas outras consequências de causas externas. Dentre os principais motivos de internação de idosos em hospitais, as doenças respiratórias e causas externas estavam entre as seis causas mais comuns em um estudo ${ }^{(17)}$. Em um estudo realizado em dois hospitais gerais, observou-se que as principais causas de internação de idosos foram as doenças circulatórias seguidas das doenças respiratórias, que foram os principais agravos na hospitalização dos sujeitos desta pesquisa ${ }^{(18)}$.

\section{CONCLUSÃO}

As UPAs, serviços de urgência de média complexidade, atendem atualmente situações e doenças que normalmente poderiam ser atendidos na UBS, fato este demonstrado pelos atendimentos classificados como pouco urgentes. Além disso, grande parte dos agravos atendidos trata-se de sintomatologias não relacionadas diretamente com patologias, passíveis de intervenção na UBS. 
Apesar da ênfase no processo de reorientação no modelo assistencial atual e da definição da APS como porta de entrada do sistema de saúde, infere-se que os usuários tendem a preferir os prontos atendimentos e hospitais para iniciar suas terapêuticas, o que ocasiona a superlotação dos serviços de média e alta complexidade, dificultando a operacionalização da rede.

Como implicações para a prática, torna-se necessário reforçar a importância da UBS como um componente essencial da RUE, na busca da organização do fluxo de atendimento, oferecendo assistência nos agravos mais comuns de saúde e a referência aos usuários quando houver a ocorrência de doenças que requeiram maior aparato tecnológico.

Como limitações, esse estudo foi realizado com a análise de dados de uma unidade de saúde do município localmente, bem como em relatórios que possuíam falhas de preenchimento. Contudo, os resultados apresentam um panorama de análise para aplicação em outras realidades, o que representa umas das recomendações para pesquisas futuras.

\section{REFERÊNCIAS}

1. Ministério da Saúde. Portaria n. 4.279, de 30 de dezembro de 2010. Estabelece diretriz para a organização da Rede de Atenção à Saúde no âmbito do Sistema Único de Saúde (SUS). Diário Oficial da União,31 dez 2010. Seção1.

2. Organização Pan-Americana da Saúde (OPAS).Organização Mundial da Saúde.Conselho Nacional de Secretários de Saúde.Mendes EV. As redes de atenção à saúde. [Internet] Brasília: OPAS; 2011 [acesso em 09 set 2015]. Disponível: http://www.conass.org.br/bibliotecav3/pdfs/redesAtencao.pdf.

3. Universidade Federal do Maranhão (UFMA), Universidade Aberta do SUS (UMA-SUS). Redes de atenção à saúde: a atenção à saúde organizada em redes. [Internet] São Luís: UFMA/UNA-SUS; 2015 [acesso em 13 out 2015]. Disponível: https://ares.unasus.gov.br/acervo/handle/ARES/2444.

4. Mansur AP, Favarato D. Mortalidade por doenças cardiovasculares no Brasil e na região metropolitana de São Paulo: atualização 2011. Arq. Bras. Cardiol. [Internet] 2012;99(2) [acesso em 02 out 2017]. Disponível: http://dx.doi. org/10.1590/S0066-782X2012005000061.

5. Secretaria Municipal de Saúde(SMS) (Curitiba). Relatório de gestão: período de janeiro a dezembro de 2016. [Internet] Curitiba: SMS; 2016 [acesso em 07 mai 2017]. Disponível: http://www.saude.curitiba.pr.gov.br/images/relatorio\%20_SARGSUS_2016\%20final\%20apos\%20reaprecia\%C3\%A7\%C3\%A3o.pdf.

6. Jorge AO, Coutinho AAP, Cavalcante APS, Fagundes AMS, Pequeno CC, do Carmo M, et al.Entendendo os desafios para a implementação da rede de atenção às urgências e emergências no Brasil: uma análise crítica. Divulgação em Saúde para Debate. [Internet] 2014;(52) [acesso em 29 mai 2015]. Disponível: http://cebes.org.br/site/wp-content/ uploads/2014/12/Divulgacao-52.pdf.

7. Torres SFS, Belisário SA, Melo EM. A rede de urgência e emergência da macrorregião norte de Minas Gerais: um estudo de caso. Saude soc. [Internet] 2015;24(1) [acesso em 02 out 2017]. Disponível: http://dx.doi.org/10.1590/S010412902015000100028.

8. Ministério da Saúde (BR). Secretaria de Atenção à Saúde. Departamento de Atenção Especializada. Manual Instrutivo da Rede de Atenção às Urgências e Emergências no Sistema Único de Saúde (SUSS). [Internet] Brasília: Ministério da Saúde; 2013 [acesso em 29 mai 2016]. Disponível: http://bvsms.saude.gov.br/bvs/publicacoes/manual_instrutivo_rede_ atencao_urgencias.pdf.

9. Garcia VM, Reis RK. Adequação da demanda e perfil de morbidade atendida em uma unidade não hospitalar de urgência e emergência. Cienc Cuid Saude. [Internet] 2014;13(4) [acesso em 02 out 2017]. Disponível: http://dx.doi. org/10.4025/cienccuidsaude.v13i4.19127.

10. Acosta AM, Lima MADS. Características de usuários frequentes de serviços de urgência: revisão integrativa. Rev. Eletr. Enf. [Internet] 2013;15(2) [acesso em 02 out 2017]. Disponível: http://dx.doi.org/10.5216/ree.v15i2.17526.

11. Ferrari R, Loyola AP, Pansani AP, Santos BL, Azeredo DB, Magalhães FAA, et al. Perfil da demanda dos usuários da clínica da família e da UPA em área com $100 \%$ de cobertura de atenção primária. Revista Eletrônica Gestão \& Saúde. [Internet] 2014;5(2) [acesso em 02 out 2017]. Disponível: http://periodicos.unb.br/index.php/rgs/article/ view/22781/16329. 
12. Acosta AM, Lima MADS. Usuários frequentes de serviço de emergência: fatores associados e motivos de busca por atendimento. Rev. Latino-Am. Enfermagem. [Internet] 2015;23(2) [acesso em 02 out 2017]. Disponível: http://dx.doi. org/10.1590/0104-1169.0072.2560.

13. Coutinho AAP, Cecílio LCO, Mota JAC. Classificação de risco em serviços de emergência: uma discussão da literatura sobre o Sistema de Triagem de Manchester. Rev. Med. Minas Gerais. [Internet] 2012;22(2) [acesso em 12 jun 2016]. Disponível: http://rmmg.org/artigo/detalhes/101.

14. Coelho MF, Goulart BF, Chaves LDP. Clinical emergencies: profile of hospital assistance. Rev. Rene. [Internet] 2013;14(1) [acesso em 12 jun 2016]. Disponível: http://www.revistarene.ufc.br/revista/index.php/revista/article/ view/89/.

15. dos Santos CTB, de Andrade LOM, Silva MJ, de Sousa MF. Percurso do idoso em redes de atenção à saúde: um elo a ser construído. Physis [Internet] 2016;26(1) [acesso em 02 out 2017]. Disponível: http://dx.doi.org/10.1590/S010373312016000100005 .

16. Kebian LVA, Acioli S. A visita domiciliar de enfermeiros e agentes comunitários de saúde da Estratégia Saúde da Família. Rev. Eletr. Enf. [Internet] 2014;16(1) [acesso em 02 out 2017]. Disponível: http://dx.doi.org/10.5216/ree. v16i1.20260.

17. Castro VC, Borghi AC, Mariano PP, Fernandes CAM, Mathias TAF, Carreira L. Perfil de internações hospitalares de idosos no âmbito do Sistema Único de Saúde. Rev. Rene. [Internet] 2013;14(4) [acesso em 15 jun 2016]. Disponível: http://www.revistarene.ufc.br/revista/index.php/revista/article/viewFile/1269/pdf.

18. Jobim EFC, de Sousa VO, Cabrera MAS. Causas de hospitalização de idosos em dois hospitais gerais pelo Sistema Único de Saúde (SUS). Acta Scientiarum. Health Sciences. [Internet] 2010;32(1) [acesso em 02 out 2017]. Disponível: http://dx.doi.org/10.4025/actascihealthsci.v32i1.5631. 